\title{
Erratum to: Multiple Articles of KSCE Journal of Civil Engineering
}

\section{The Publisher}

Erratum to: KSCE Journal of Civil Engineering (2020) 24(2)

This erratum is to notify a mismatch of DOIs between the items uploaded in Springer web page and the final manuscripts published in Volume 24, Issue 2 (Feb. 2020). Due to a technical error, incorrect DOIs were used in the Springer web page. The DOIs in the published issue are correct. Please find the correct DOIs below:

\section{Errata:}

Incorrect DOIs in Springer web page:

$10.1007 / \mathrm{s} 12205-020-0217-9$

$10.1007 / \mathrm{s} 12205-020-2201-9$

$10.1007 / \mathrm{s} 12205-020-0824-5$

$10.1007 / \mathrm{s} 12205-020-0850-3$

$10.1007 / \mathrm{s} 12205-020-1080-4$

$10.1007 / \mathrm{s} 12205-020-0996-\mathrm{z}$

$10.1007 / \mathrm{s} 12205-020-1837-9$

$10.1007 / \mathrm{s} 12205-020-0937-\mathrm{x}$

$10.1007 / \mathrm{s} 12205-020-0978-1$

Correct DOIs in published manuscripts:

10.1007/s12205-019-0217-9

$10.1007 / \mathrm{s} 12205-019-2201-9$

$10.1007 / \mathrm{s} 12205-019-0824-5$

$10.1007 / \mathrm{s} 12205-019-0850-3$

$10.1007 / \mathrm{s} 12205-019-1080-4$

$10.1007 / \mathrm{s} 12205-019-0996-\mathrm{Z}$

$10.1007 / \mathrm{s} 12205-019-1837-9$

$10.1007 / \mathrm{s} 12205-019-0937-\mathrm{x}$

$10.1007 / \mathrm{s} 12205-019-0978-1$

We apologize for the inconvenience. 